\title{
New multiple matched filter: design and experimental realization
}

\author{
Santiago Vallmitjana, Salvador Bosch, Ignacio Juvells, and Domenec Ros
}

\begin{abstract}
A method of making a multiple matched filter which allows the recognition of different characters in successive planes in simple conditions is proposed. The generation of the filter is based on recording on the same plate the Fourier transforms of the different patterns to be recognized, each of which is affected by different spherical phase factors because the patterns have been placed at different distances from the lens. This is proved by means of experiments with a triple filter which allows satisfactory recognition of three characters.
\end{abstract}

\section{Introduction}

One of the most interesting optical processing techniques is the matched filtering of an input signal. To achieve the pattern recognition, this technique performs the optical correlation between the input and pattern. The most common method of doing this is to use the VanderLugt technique based on the Fourier hologram of the pattern to be detected. ${ }^{1}$

To detect different characters it is possible to use a multiple matched filter instead of a set of individual filters. This can be done by recording the single interference patterns on a sole holographic plate. Some different methods for registering the multiple matched filter can be found in Refs. 2-5.

In the VanderLugt technique, a common setup for obtaining the filter of a pattern is to place the object in the front focal plane of a lens and register the interferences obtained in its back focal plane between a reference tilted plane wave and the Fourier transform distribution produced by the lens. The filtering process is performed in an optical setup consisting of two lenses, each transforming the complex amplitude from the front to the back focal plane. However, other configurations have already been proposed, both for the filter construction ${ }^{6}$ and the filtering process. ${ }^{7}$

In light of these ideas, in the present work we allow the distance from the object to the lens in the registering process and the distance between lenses when filtering to be different from the focal ones. Then the correlation is also obtained in a plane that is not the back focal plane of the second lens. Its position depends on all the distances involved in the registering and filtering processes.

All authors are with University of Barcelona, Diagonal 647, 08028 Barcelona, Spain; D. Ros is in the Department of Physical Medicine, the other authors are in the Optics Department.

Received 17 May 1986.

0003-6935/86/234473-03\$02.00/0.

(C) 1986 Optical Society of America.
Consequently, it is possible to construct a multiple matched filter by using different registering positions for each character so that the corresponding correlations will be obtained in different planes. We analyze a special case of particular interest, and, for this, we construct a multiple matched filter able to detect three different characters, and we show the results.

\section{Method}

Let us assume that a plane wave of unit amplitude illuminates an object with amplitude transmittance $t_{0}\left(x_{0}, y_{0}\right)$ placed at a distance $d_{F}$ from a lens of focal length $f_{F}$ [Fig. 1(a)]. The pattern obtained in the back focal plane of the lens when the interference with a tilted plane wave is registered can be used to obtain a filter whose transmittance is ${ }^{8}$

$$
\begin{aligned}
& t_{F}\left(x_{F}, y_{F}\right)= r_{0}^{2}+\frac{1}{\lambda^{2} f_{F}^{2}}\left|T_{0}\left(\frac{x_{F}}{\lambda f_{F}}, \frac{y_{F}}{\lambda f_{F}}\right)\right|^{2} \\
&(\S)+\frac{r_{0}}{\lambda f_{F}} \exp \left(-i 2 \pi \alpha y_{F}\right) \exp \left[-i \frac{k}{2 f_{F}}\left(1-\frac{d_{F}}{f_{F}}\right)\left(x_{F}^{2}+y_{F}^{2}\right)\right] \\
& \times T_{0}^{*}\left(\frac{x_{F}}{\lambda f_{F}}, \frac{y_{F}}{\lambda f_{F}}\right)
\end{aligned}
$$$$
\begin{aligned}
(\S \S)+\frac{r_{0}}{\lambda f_{F}} & \exp \left(i 2 \pi \alpha y_{F}\right) \exp \left[i \frac{k}{2 f_{F}}\left(1-\frac{d_{F}}{f_{F}}\right)\left(x_{F}^{2}+y_{F}^{2}\right)\right] \\
& \times T_{0}\left(\frac{x_{F}}{\lambda f_{F}}, \frac{y_{F}}{\lambda f_{F}}\right)
\end{aligned}
$$

$$
(\alpha=\sin \theta / \lambda) .
$$

We can use this filter to process an input signal $g\left(x_{0}, y_{0}\right)$. When illuminating with a plane wave whose amplitude is $A$, assume that $g\left(x_{0}, y_{0}\right)$ is at a distance $d_{0}$ from a lens of focal length $f_{F}$ and that a second lens of focal length $f_{C}$ is placed at a distance $d_{R}$ from the back focal plane of the first lens [Fig. 1(b)].

Since the lens used for registering the filter had a focal length $f_{F}$, if we place the filter in the back focal plane of the first lens, the transmitted amplitude in this plane will be 


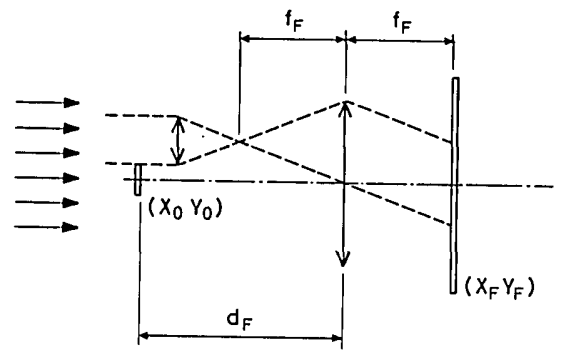

(a)

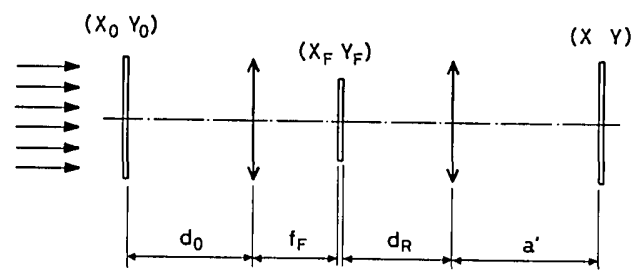

(b)

Fig. 1. Experimental setup: (a) arrangement for the recording of the filter; (b) filtering arrangement.

$$
\begin{aligned}
t\left(x_{F}, y_{F}\right)= & t_{F}\left(x_{F}, y_{F}\right) \frac{1}{\lambda f_{F}} \\
& \times \exp \left[i \frac{k}{2 f_{F}}\left(1-\frac{d_{0}}{f_{F}}\right)\left(x_{F}^{2}+y_{F}^{2}\right)\right] G\left(\frac{x_{F}}{\lambda f_{F}}, \frac{y_{F}}{\lambda f_{F}}\right),
\end{aligned}
$$

where $G$ is the Fourier transform of $g$.

From (§), putting together the quadratic phase terms and neglecting constants, we have

$\exp \left(-i 2 \pi \alpha y_{F}\right) \exp \left\{i \frac{k}{2 f_{F}}\left(x_{F}^{2}+y_{F}^{2}\right)\right.$

$$
\left.\times\left[\left(1-\frac{d_{0}}{f_{F}}\right)-\left(1-\frac{d_{F}}{f_{F}}\right)\right]\right\} G \cdot T_{0}^{*} .
$$

The second exponential can be interpreted as a spherical wave that illuminates an object $\mathrm{G} \cdot \mathrm{T}_{0}^{*}$ placed in the filtering plane. The point source producing the spherical wave would be on-axis at a distance $b_{1}$ in front of the object, where $b_{1}$ is calculated from

$$
\frac{1}{b_{1}}=\frac{1}{f_{F}}\left\{\left(1-\frac{d_{0}}{f_{F}}\right)-\left(1-\frac{d_{F}}{f_{F}}\right)\right\}=\frac{d_{F}-d_{0}}{f_{F}^{2}} .
$$

In these conditions, ${ }^{7,9}$ the corresponding Fourier transform, i.e., the cross-correlation $g * t_{0}$ (affected by a quadratic phase term) will be obtained at a certain distance $a_{1}^{\prime}$ from the lens, being $a_{1}=b_{1}+d_{R}$ and $1 / a_{1}+$ $1 / a_{1}^{\prime}=1 / f_{C} ;$ then

$$
a_{1}^{\prime}=f_{C} \frac{f_{F}^{2}+d_{R}\left(d_{F}-d_{0}\right)}{f_{F}^{2}+\left(d_{R}-f_{C}\right)\left(d_{F}-d_{0}\right)} .
$$

The scale is given by

$$
\frac{1}{\lambda f_{C}} \frac{f_{F}^{2}+\left(d_{R}-f_{C}\right)\left(d_{F}-d_{0}\right)}{f_{F}^{2}} .
$$

Similarly, from $(\S \S)$ we can see that the convolution $g * t_{0}$ is obtained at $a_{2}^{\prime}$ given by

$$
a_{2}^{\prime}=f_{C} \frac{f_{F}^{2}+d_{R}\left[2 f_{F}-\left(d_{F}+d_{0}\right)\right]}{f_{F}^{2}+\left(d_{R}-f_{C}\right)\left[2 f_{F}-\left(d_{F}+d_{0}\right)\right]} .
$$

\section{Multiple Matched Filter}

Equation (1) gives the position of the plane where the correlation appears depending on the different distances involved in the registering and filtering processes. From this, a multiple matched filter can be constructed: by changing some parameters in the registration of the filter, the detection of the different patterns recorded on it is achieved in different planes.

Taking into account (2), we can see that $d_{R}=f_{C}$ implies that the scale factor is always $1 /\left(\lambda f_{C}\right)$. In this case Eq. (1) depends only on $d_{F}$ and $d_{0}$. Moreover, if $d_{0}$ $=f_{F}$, this yields

$$
a_{1}^{\prime}=\frac{f_{C}^{2}}{f_{F}^{2}} d_{F}+f_{C}\left(1-\frac{f_{C}}{f_{F}}\right) ;
$$

that is, if we set the filtering distances to be the focal ones (standard setup), allowing only the registering value $d_{F}$ to change for each different pattern, the position $a_{1}^{\prime}$ of the plane where the correlation appears varies linearly with respect to $d_{F}$ and the scale factor remains unchanged. Furthermore, the slope of the straight line [Eq. (4)] is $f_{C}^{2} / f_{F}^{2}$; so if we use $f_{C}>f_{F}$ small differences in registration correspond to bigger distances between correlation planes; i.e., there will be no overlapping between correlations.

In this way, we can construct a multiple matched filter for different characters by simply placing them in different positions near the front focal plane in the registration process. Using this filter in a standard character recognition setup, we can obtain the correlations between the input signal and the different characters by considering the corresponding image planes. Moreover, the scale of this correlation remains the same for all the characters.

\section{Experimental Method}

\section{A. Input Signal and Pattern to be Detected}

As a test signal $g\left(x_{0}, y_{0}\right)$ we used a matrix formed by nine characters [Fig. 2(a)]. It was registered on a high contrast film, and its dimensions were $6.5 \times 6.5 \mathrm{~mm}$, the letters being $1.5 \mathrm{~mm}$ high. The aim was to detect three different letters: $R, K$, and $W$.

\section{B. Recording the Multiple Filter}

We used an optical processor setup where a plane wave illuminated the pattern. The light source was a 7 - $\mathrm{mW}$ laser. The Fourier lens is a well-corrected doublet of $f_{F}=50 \mathrm{~cm}$ and $\phi=f / 7.14$. The multiple filter was registered by the incoherent recording method to avoid vignetting effects that can arise in coherent registering, although the efficiency is decreased. ${ }^{2}$

The film containing each target to be detected was introduced into a liquid gate to avoid phase effects. It is important, therefore, to check the position of the central peak in the Fourier plane because the glass 


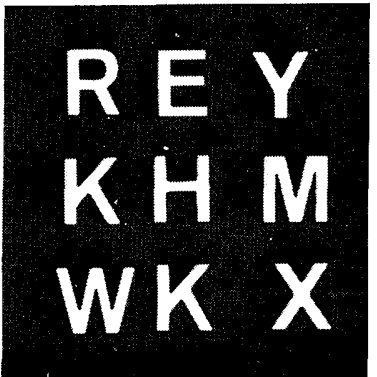

a

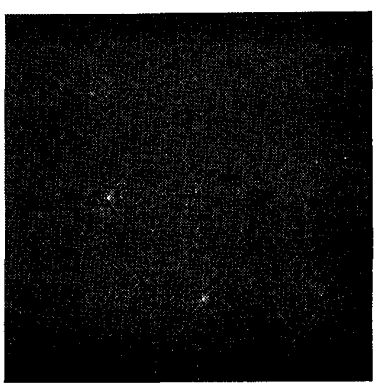

C

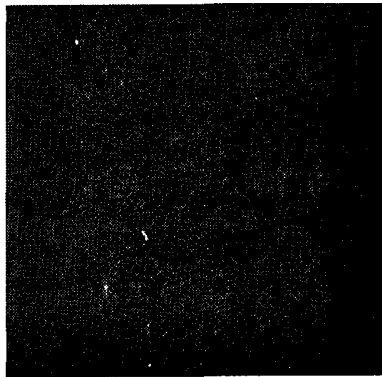

b

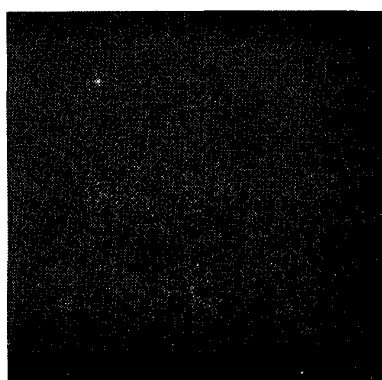

d
Fig. 2. Successive detection of characters $W, K$, and R: (a) input signal; (b) detection of $\mathrm{W}$ at a distance of 154.5 from the second lens; (c) detection of $\mathrm{K}$ at $104 \mathrm{~cm}$; (d) detection of $R$ at $70.5 \mathrm{~cm}$.

plates can easily provoke a prism effect that translates the diffraction pattern to be registered from one target to another. It is also important to check the perfect relative orientation between the characters and input signal.

The Fourier hologram was registered by placing the three characters at respective distances $d_{F}(W)=55.8$ $\mathrm{cm}, d_{F}(K)=47.3 \mathrm{~cm}$, and $d_{F}(R)=41.3 \mathrm{~cm}$ from the Fourier lens. The holographic plate was developed and bleached using well-known techniques. ${ }^{10}$

\section{Pattern Detection}

The input signal $g\left(x_{0}, y_{0}\right)$ was placed in the front focal plane of the first lens $\left(f_{F}=50 \mathrm{~cm}\right)$ and the filter in its back focal plane which coincides with the front focal plane of the second lens $\left(f_{C}=120 \mathrm{~cm}, \phi=f / 16\right)$. In this case Eq. (4) gives (in centimeters)

$$
a_{1}^{\prime}=5.76 d_{F}-168 \text { (cross-correlation) }
$$

and Eq. (3)

$$
a_{2}^{\prime}=-5.76 d_{F}+408 \text { (convolution). }
$$

The slope 5.76 means that small changes in the position in the filter registration process cause significant changes in the location of the correlation plane with the result that erroneous detections are avoided.

When changing the output plane, we can note the progressive appearance and disappearance of the correlation maxima as we consider zones near the corresponding positions predicted by Eq. (1).

In Table I the theoretical distances for the crosscorrelations are given as well as the experimental results that we obtained (positions where the correlation
Table I. Position of the Correlation Plane ${ }^{a}$

\begin{tabular}{cccr}
\hline Character & $d_{F}$ & $a_{1}^{\prime}$ & \multicolumn{1}{c}{$e$} \\
\hline $\mathrm{R}$ & 41.3 & 69.8 & 70.5 \\
$\mathrm{~K}$ & 47.3 & $\mathbf{1 0 4 . 4}$ & 104.0 \\
$\mathrm{~W}$ & 55.8 & 153.4 & 154.5 \\
\hline
\end{tabular}

${ }^{a} d_{F}$, filter registering distance; $a_{1}^{\prime}$, theoretical position; $e$, experimentally measured position.

peak was visually sharper). Figures $2(b),(c)$, and (d) show these results.

\section{Conclusions}

When a matched filter is registered by placing the object out of the front focal plane of the lens and then used in a standard filtering setup (input and filter in focal planes), the position of the correlation plane varies linearly with respect to the registering displacement. This permits us to construct multiple filters by placing the different patterns in different planes in the registration. By suitable choice of the focal lengths of the lenses it is possible to obtain a very good resolution in the detection: small changes in the registering position give greater differences in correlation planes. (This means no erroneous detections.)

The experimental results show that it is possible to obtain without difficulty a triple filter that permits us to detect perfectly the characters, even when two of them are similar (letters $\mathrm{K}$ and $\mathrm{R}$ ).

This work has been supported in part by Comisión Asesora de Investigación Cientifica y Técnica, $r / N$ 3300-83.

\section{References}

1. A. B. VanderLugt, "Signal Detection by Complex Spatial Filtering," IEEE Trans. Inf. Theory IT-10, 139 (1964).

2. J. T. LaMacchia and C. J. Vincelette, "Comparison of the Diffraction Efficiency of Multiple Exposure and Single Exposure Holograms," Appl. Opt. 7, 1857 (1968).

3. A. B. VanderLugt, F. B. Rotz, and A. Klooster, Jr., "CharacterReading by Optical Spatial Filtering," in Optical and Electrooptical Information Processing, J. T. Tippett, D. A. Berkowitz, L. C. Clapp, C. Koester, and A. Vanderburg, Jr., Eds. (MTT Press, Cambridge, 1965), Chap. 7, pp. 125-141.

4. J. C. Vienot, J. Bulabois, and L. R. Guy, "Three Dimensional Object Recognition in Real Time by Multiplex Spatial Filtering," Opt. Commun. 2, 431 (1971).

5. C. Ferreira, P. Andrés, E. Bonet, A. Pons, and M. Aguilar, "Character Recognition: a New Multiple Filter," Opt. Commun. 47, 177 (1983).

6. A. VanderLugt, "Practical Considerations for the Use of Spatial Carrier-Frequency Filters,” Appl. Opt. 5, 1760 (1966).

7. A. VanderLugt, "Operational Notation for the Analysis and Synthesis of Optical Data-Processing Systems," Proc. IEEE 54, 1055 (1966).

8. J.W. Goodman, Introduction to Fourier Optics (McGraw-Hill, New York, 1968), Chap. 5.

9. G. Breuer, "A Formal Representation of Abbe's Theory of Microscopic Image Formation," Opt. Acta 31, 661 (1984); formula (26) corrected.

10. J. Oliva, M. Pardo, and J. A. Quintana, "High SNR in Bleached Silver-Halide Holography," Appl. Opt. 21, 171 (1982). 